\title{
Intégration des concepts de la fiabilité et de l'optimisation en conception de prothèses orthopédiques : Application sur la conception de la prothèse de hanche
}

\section{Integration of reliability and optimization conception into orthopedic prosthesis design: Application on hip prosthesis}

\author{
Kharmanda G. ${ }^{1}$, El-Hami A. ${ }^{2}$, Ibrahim M-H. ${ }^{3}$ \\ ${ }^{1}$ Department of Biomedical Engineering, Lund University, Lund, Sweden, ghias.kharmanda@bme.Ith.se \\ ${ }^{2}$ Laboratoire d'optimisation et fiabilité en mécanique des structures, INSA de Rouen, abdelkhalak.elhami@insa-rouen.fr \\ ${ }^{3}$ Ecole d'ingénieurs de l'université d'Angers, mohamed.ibrahim@univ-angers.fr
}

RÉSUMÉ. L'intégration des concepts de l'optimisation et de la fiabilité en conception de prothèses se retrouve dans un espace scientifique commun pour mieux comprendre les différents effets biomécaniques. Dans ce travail, l'intégration est effectuée en conception des prothèses orthopédiques pour augmenter la performance et également garantir un niveau de fiabilité cible. Une application numérique sur la conception d'une prothèse de hanche a été sélectionnée en considérant l'incertitude sur les propriétés mécaniques des matériaux.

ABSTRACT. The integration of optimization and reliability concepts into prosthesis design allows to understand the different biomechanical effects. In this work, the integration is carried out in the orthopedic prosthesis design in order to increase the performance and also to guarantee a required reliability level. An numerical application on the hip prosthesis design is selected considering the mechanical material uncertainties.

MOTS-CLÉS. Conception de prothèse de hanche, Optimisation structurale, Analyse de fiabilité, Optimisation fiabiliste de conception, Mode multiple de défaillance.

KEYWORDS. Hip prosthesis design, Structural optimization, Reliability analysis, Reliability-Based Design Optimization, Multiple failure modes.

\section{Introduction}

Les modifications des caractéristiques d'un implant peuvent conduire à des différences significatives du comportement clinique à court ou à long terme. L'évaluation d'un implant, en termes de contrôle de qualité ne peut se faire en définitive que par l'investigation clinique, c'est justement à cause de cet aspect que le domaine de conception des prothèses ne peut jamais être déterministe. En effet, l'intégration de l'analyse de fiabilité permet simplement d'estimer la stabilisation de la prothèse [KHA 15]. De plus, l'intégration de l'optimisation structurale déterministe permet seulement d'augmenter la performance de la prothèse [KHA 16] sans prendre en compte l'incertitude. Cependant, l'intégration de la fiabilité et de l'optimisation (optimisation fiabiliste) en conception de prothèses est une outil indispensable pour augmenter la performance des prothèses et respecter un niveau requis de fiabilité. Dans ce travail, on applique le couplage d'optimisation fiabiliste en conception d'une prothèse de hanche en considérant l'incertitude sur les propriétés mécaniques des matériaux utilisés puisque la densité de l'os dépend de plusieurs facteurs (âge, nourriture ...). De plus, après l'opération chirurgicale, cette densité peut être diminuée. Deux formulations sont utilisées pour relier les propriétés mécaniques des tissues de l'os.

\section{Optimisation et fiabilité}

Le modèle de l'intégration de la fiabilité en optimisation est appelé optimisation fiabiliste (ReliabilityBased Optimization). En général, la méthode classique de l'optimisation fiabiliste consiste à effectuer deux sous problèmes dans deux espaces différents : espaces physique et normé (figure 1) : 
Problème d'optimisation : Ce problème est effectué en intégrant la contrainte de fiabilité :

$\begin{array}{cl}\min _{\mathbf{x}} & f(\mathbf{x}) \\ \text { sous } & g_{k}(\mathbf{x}) \leq 0 \quad, k=1, \ldots, K \\ & \beta(\mathbf{x}, \mathbf{u}) \geq \beta_{c}\end{array}$

où $\mathbf{x}$ et $\mathbf{u}$ sont les vecteurs de variables déterministes et normées. $f(\mathbf{x})$ est la fonction objectif à minimiser (coût, poids, ...) sous les contraintes d'optimisation $g_{k}(\mathbf{x}) \leq 0$ et la contrainte de fiabilité $\beta(\mathbf{x}, \mathbf{u}) \geq \beta_{c}$, $\beta(\mathbf{x}, \mathbf{u})$ est l'indice de fiabilité de la structure et $\beta_{c}$ est l'indice de fiabilité cible.

Problème de fiabilité : Ce problème peut être effectué sous la minimisation suivante :

$$
\begin{array}{ll}
\min _{\mathbf{u}} & d(\mathbf{u})=\sqrt{\sum u_{i}^{2}} \\
\text { sous } & H(\mathbf{x}, \mathbf{u}) \leq 0
\end{array}
$$

La résolution des deux problèmes imbriqués (1 et 2) peut conduire a plusieurs difficultés comme temps de calcul, convergence, optimum local...

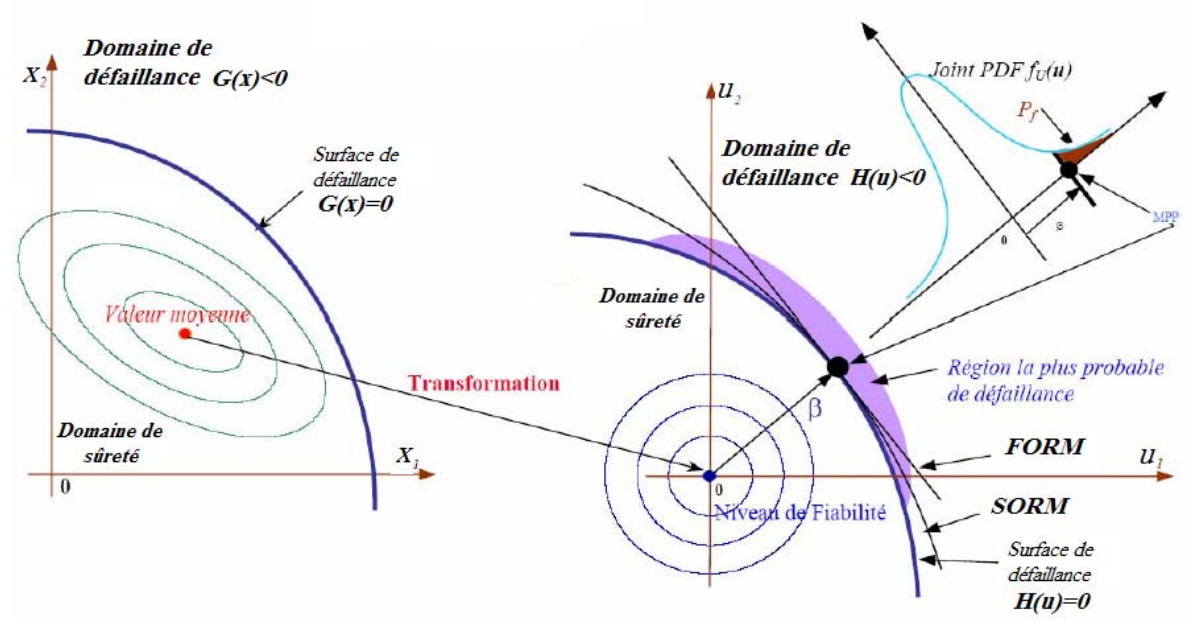

Figure 1. Espace physique et celui normé

Plusieurs méthodes sont développées pour palier les difficultés rencontrées [KHA 10]. Parmi ces méthodes, on a la méthode hybride où le processus de conception est effectué d'une façon efficace dans un espace de conception hybride regroupant tout les variables déterministes et normées.
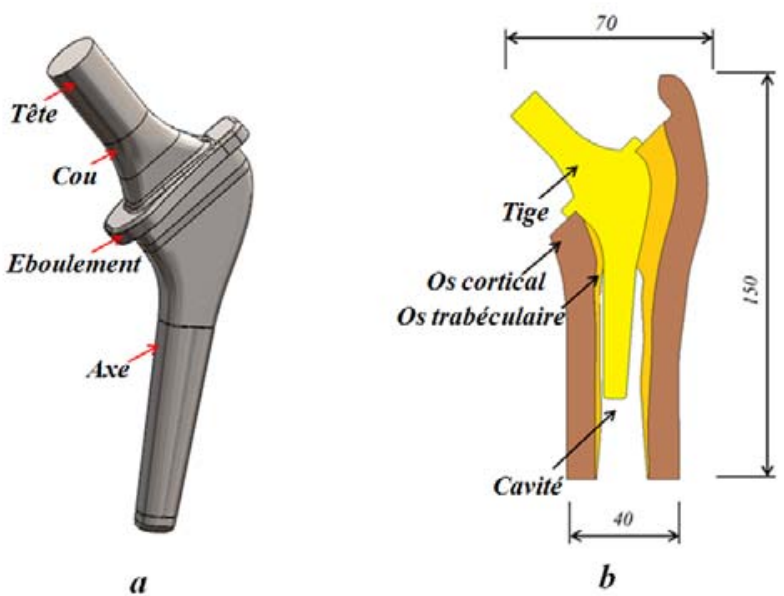

Figure 2. a) Modèle géométrique de la tige étudié en $3 D$ et b) interface implant-os en 2D 


\section{Application numérique de la prothèse de hanche}

La tige de la prothèse de hanche est entouré par les tissues de l'os. La modélisation géométrique est compliquée à effectuer en 3D. La figure 2 a montre la tige à étudier en 3D. Cependant, pour simplifier la programmation ou l'implémentation, on modélise la structure composée en 2D (figure 2b) pour effectuer l'analyse de fiabilité et l'optimisation fiabiliste de conception. Les tissus osseux (cortical et spongieux) et le métal (titane) sont considérées homogènes et iso-tropiques avec les propriétés mécaniques suivantes : $E_{\text {Cor }}=19.7 \mathrm{GPa}, v_{\text {Cor }}=0.33, E_{\text {Trab }}=5.6 \mathrm{GPa}, v_{\text {Trab }}=0.33, E_{\text {Met }}=120 \mathrm{GPa}, v_{\text {Met }}=0.36$. Les trois cas du chargement [BEA 90] sont considérées comme dans la figure 2 (unijambiste position (L1), mouvement d'enlèvement (L2) et mouvement d'adduction (L3)).
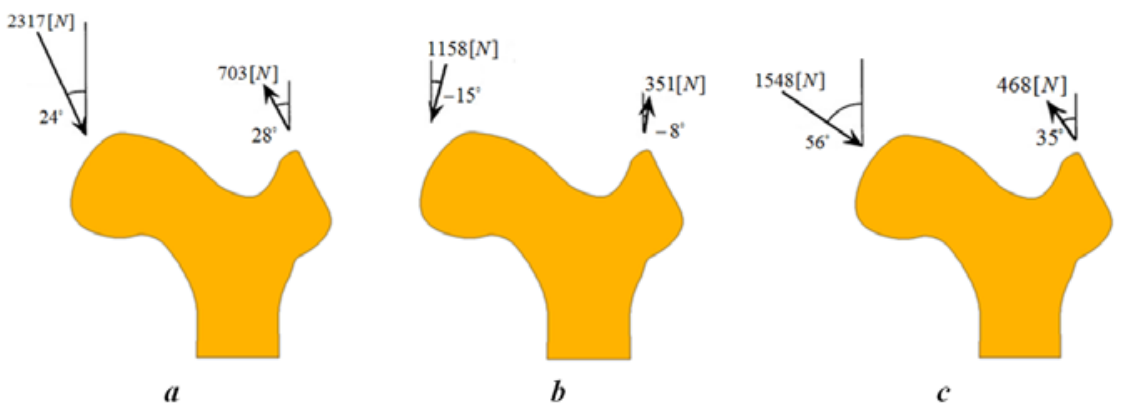

Figure 3. Conditions aux limites: $L 1$, $L 2$ et $L 3$

Dans le cas étudié, la densité de l'os se change selon plusieurs facteurs; ce qui conduit au changement des propriétés mécaniques. Deux modèles peuvent être considérés pour relier les propriétés mécaniques de l'os : le modèle de Keller [KEL 94] :

$$
\sigma_{T}=117 R_{T / C}\left(\frac{E}{10500}\right)^{\left(\frac{1.93 \pm 0.04}{2.57 \pm 0.04}\right)}
$$

et le modèle de Kharmanda [KHA 15] :

$$
\sigma_{T}=129.5 R_{T / C}\left(\frac{E}{11142}\right)^{0.94}
$$

Pour l'os cortical, on considère le taux Tension/Compression est égale à $R_{T / C}=0.7$ tandis que pour l'os spongieux (trabéculaire), ce taux est de $R_{T / C}=1$ [DOB 04].

\subsection{Simulation directe}

Pour déterminer les cas critiques, on fait une simulation directe du point courant. Le tableau 3.1 présente les valeurs maximales des contraintes de von-Mises.

\begin{tabular}{|l|c|c|c|}
\hline \multicolumn{1}{|c|}{ Paramètres } & L1 & L2 & L3 \\
\hline$\sigma_{\max }^{\text {Metal }}(\mathbf{M P a})$ & 167.96 & 103.56 & 113.33 \\
\hline$\sigma_{\max }^{\text {Cor }}(\mathbf{M P a})$ & 129.34 & 90.32 & $\underline{143.97}$ \\
\hline$\sigma_{T}^{\text {Cor_Keller }}(\mathbf{M P a})$ & \multicolumn{3}{|c|}{$[129.17 \div 133.68]$} \\
\hline$\sigma_{T}^{\text {Cor_Kharmanda }}(\mathbf{M P a})$ & \multicolumn{3}{|c|}{154.89} \\
\hline$\sigma_{\max }^{\text {Trab }}(\mathbf{M P a})$ & 44.30 & 23.47 & 62.08 \\
\hline$\sigma_{T}^{\text {Trab_Keller }}(\mathbf{M P a})$ & \multicolumn{3}{|c|}{$[71.72 \div 74.22]$} \\
\hline$\sigma_{T}^{\text {Trab_Kharmanda }}(\mathbf{M P a})$ & \multicolumn{3}{|c|}{67.83} \\
\hline
\end{tabular}

Tableau 3.1. Valeurs maximales des contraintes de von-Mises d'une simulation directe 
On peut noter que la contrainte maximale de von-Mises pour le troisième cas du chargement L3 a dépassé la limite élastique pour l'os cortical en considérant la formulation de Keller [KEL 94]. Cependant, cette valeur est inférieure de la limite élastique en considérant la formulation de Kharmanda [KHA 15].

\subsection{Analyse de fiabilité}

Dans cette section, l'analyse de fiabilité de la tige avec l'os est réalisée en considérant les deux formulations (Keller [KEL 94] et Kharmanda [KHA 15]). Ces deux formulations sont intégrées dans un algorithme de calcul de l'indice de fiabilité. Le tableau 3.2 montre les résultats de l'analyse de fiabilité de la tige en utilisant les deux formulations pour le cas d'un seul scenario de défaillance (cortical ou trabéculaire) et pour le cas (système) de deux scenarios de défaillance (cortical et trabéculaire).

\begin{tabular}{|c|c|c|c|c|}
\hline \multirow{2}{*}{ Modèles } & Paramètres & L1 & L2 & L3 \\
\cline { 2 - 5 } Modèle de Keller & $\beta_{\text {Cor }}^{\text {Keller }}$ & $\underline{0.02}$ & 3.84 & Défaillance \\
\cline { 2 - 5 } & $\beta_{\text {Trab }}^{\text {Keller }}$ & 5.21 & 7.04 & Défaillance \\
\cline { 2 - 5 } & $\beta_{\text {Sys }}^{\text {Keller }}$ & 5.21 & 8.54 & Défaillance \\
\hline \multirow{2}{*}{ Modèle de Kharmanda } & $\beta_{\text {Cor }}^{\text {Kharmanda }}$ & $\underline{2.82}$ & 4.38 & $\underline{1.71}$ \\
\cline { 2 - 5 } & $\beta_{\text {Trab }}^{\text {Kharmanda }}$ & 4.18 & 6.64 & $\underline{1.21}$ \\
\cline { 2 - 5 } & $\beta_{\text {Sys }}^{\text {Kharmanda }}$ & 4.27 & 8.05 & $\underline{1.29}$ \\
\hline
\end{tabular}

Tableau 3.2. Résultats de l'analyse de fiabilité de la tige en utilisant les deux formulations

Après avoir effectuée l'analyse de fiabilité, on trouve que pour le troisième cas du chargement L3, l'utilisation de la formulation de Keller conduit toujours au cas de défaillance tandis que l'utilisation de la formulation optimisée de Kharmanda conduit aux niveaux de fiabilité faibles. Pour cela, l'optimisation fiabiliste de conception est indispensable pour améliorer le niveau de fiabilité en modifiant la géométrie de la structure.

\subsection{Optimisation fiabiliste}

Les dimensions $\mathrm{A}$, $\mathrm{B}$ et $\mathrm{C}$ sont considérées comme des variables déterministes et leurs valeurs moyennes sont classées dans un vecteur déterministe $\mathbf{x}=\left\{m_{A}, m_{B}, m_{C}\right\}$. Les propriétés mécaniques représentent les variables aléatoires leurs valeurs sont classées dans le vecteur aléatoire $\mathbf{y}=\left\{E_{C o r}, E_{T r a b}, E_{\text {Met }}, v_{C o r}, V_{\text {Trab }}, v_{\text {Met }}\right\}$. En utilisant la méthode hybride [KHA 10], le problème d'optimisation fiabiliste peut s'écrire sous la forme suivante :

$$
\begin{aligned}
\min & : F(\mathbf{x}, \mathbf{y})=\sigma_{\max }^{\text {Cor }}(\mathbf{x}, \mathbf{y}) \cdot \sigma_{\max }^{\text {Trab }}(\mathbf{x}, \mathbf{y}) \cdot d_{\beta}(\mathbf{x}, \mathbf{y}) \\
\text { sous } \quad & : d_{\beta}(\mathbf{x}, \mathbf{y})=\beta_{c} \\
& : \sigma_{\max }^{\text {Cor }}(\mathbf{x}, \mathbf{y})-\sigma_{y}^{\text {Cor }}(\mathbf{x}, \mathbf{y}) \leq 0 \\
& : \sigma_{\max }^{\text {Trab }}(\mathbf{x}, \mathbf{y})-\sigma_{y}^{\text {Trab }}(\mathbf{x}, \mathbf{y}) \leq 0 \\
\text { et } \quad: & \sigma_{\max }^{\text {Met }}(\mathbf{x}, \mathbf{y})-\sigma_{y}^{\text {Met }}(\mathbf{x}, \mathbf{y}) \leq 0
\end{aligned}
$$

où $F(\mathbf{x}, \mathbf{y})$ est la forme multiplicative des fonctions objectifs à minimiser. $\sigma_{\max }^{\text {cor }}(\mathbf{x}, \mathbf{y})$ et $\sigma_{y}^{\text {cor }}$ sont respectivement la contrainte maximale de von-Mises et la limite élastique en tension (équation 4) du tissu cortical. $\sigma_{\max }^{\text {Trab }}(\mathbf{x}, \mathbf{y})$ et $\sigma_{y}^{\text {Trab }}$ sont respectivement la contrainte maximale de von-Mises et la limite élastique en tension (équation 4) du tissu trabéculaire. $\sigma_{\max }^{\text {Met }}(\mathbf{x}, \mathbf{y})$ et $\sigma_{y}^{\text {Met }}$ sont respectivement la contrainte maximale de von-Mises et la limite élastique du métal de la tige. Le problème est de minimiser les contraintes maximales de von-Mises des tissus osseux et l'indice de fiabilité en respectant un niveau de 
fiabilité requis $\left(\beta_{c}=3\right)$ et les valeurs maximales des contraintes de von-Mises qui ne doivent pas dépasser les limites élastiques des composants. Le tableau 3.3 montre les résultats de l'optimisation structurale et fiabiliste de la tige. Selon les résultats, on peut noter que pour le cas trois du chargement $\mathrm{L} 3$, on respecte le niveau demandé de fiabilité $\left(d_{\beta}=\beta_{c}=3\right)$.

\begin{tabular}{|l|c|c|c|}
\hline Paramètres & L1 & L2 & L3 \\
\hline$E_{\text {Cor }}(\mathbf{M P a})$ & 16490 & 11201 & 18436 \\
\hline$E_{\text {Trab }}(\mathbf{M P a})$ & 3459.6 & 1929.4 & 4647.4 \\
\hline$E_{\text {Met }}(\mathbf{M P a})$ & 118720 & 119960 & 100700 \\
\hline$v_{\text {Cor }}$ & 0.32800 & 0.31808 & 0.31709 \\
\hline$v_{\text {Trab }}$ & 0.32696 & 0.32991 & 0.30559 \\
\hline$v_{\text {Met }}^{\text {Met }}(\mathbf{M P a})$ & 0.34469 & 0.34908 & 0.30097 \\
\hline$\sigma_{\max }^{\text {Mar }}$ & 171.38 & 123.86 & 114.94 \\
\hline$\sigma_{\max }^{\text {Cor }}(\mathbf{M P a})$ & 130.13 & 90.281 & 144.98 \\
\hline$\sigma_{T}^{\text {Cor }}(\mathbf{M P a})$ & 131.04 & 91.098 & 145.53 \\
\hline$\sigma_{\max }^{\text {Trab }}(\mathbf{M P a})$ & $\underline{42.160}$ & $\underline{23.935}$ & $\underline{57.028}$ \\
\hline$\sigma_{C}^{\text {Trab }}(\mathbf{M P a})$ & $\underline{43.13}$ & $\underline{24.913}$ & $\underline{56.925}$ \\
\hline$\beta$ & 4.18 & 7.86 & $\mathbf{3 . 0 0}$ \\
\hline$P_{f}$ & $1.5 \times 10^{-5}$ & $1.9 \times 10^{-16}$ & $1.3 \times 10^{-3}$ \\
\hline
\end{tabular}

Tableau 3.3. Résultats de l'optimisation structurale et fiabiliste de la tige

La configuration de la tige a été modifiée pour respecter le niveau requis de fiabilité. Les figures $4 \mathrm{a}$ et $\mathrm{b}$ montrent les configurations initiale et optimale de la tige avec les tissus osseux, respectivement.
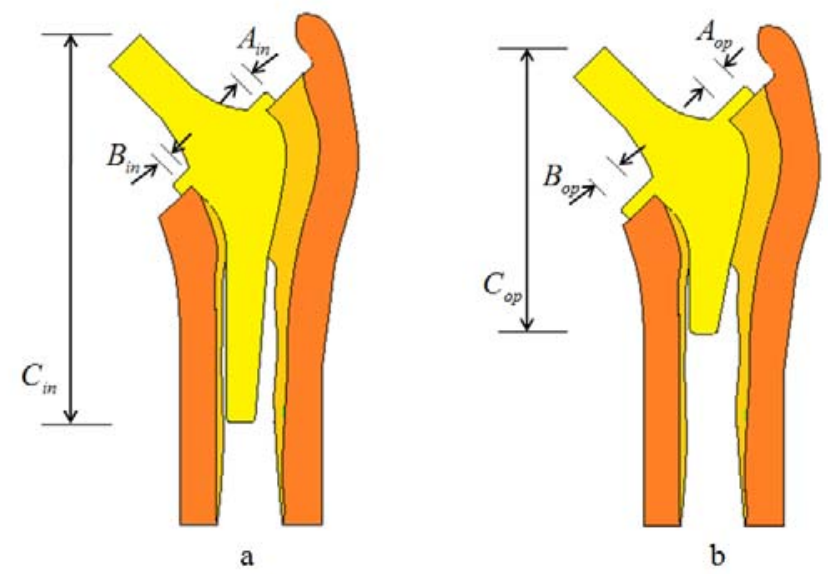

Figure 4. a) Configuration initiale et b) configuration optimale

$\mathrm{Au}$ point initial, les composantes du vecteur des paramètres d'entrée sont : $\mathrm{A}=4.00 \mathrm{~mm}, \mathrm{~B}=3.95 \mathrm{~mm}$ et $\mathrm{C}=114.33 \mathrm{~mm}$. Au point optimal, les composantes du vecteur des paramètres d'entrée sont : $\mathrm{A}=13.88 \mathrm{~mm}, \mathrm{~B}=13.62 \mathrm{~mm}$ et $\mathrm{C}=84.39 \mathrm{~mm}$. La configuration optimale représente une structure plus fiable que la configuration initiale et également en respectant un niveau cible de fiabilité.

\section{Conclusion}

Dans ce travail, l'intégration de l'optimisation et de la fiabilité en conception de prothèses orthopédiques a été réalisée d'une façon très simple avec un petit nombre de variables pour comprendre la stratégie développée. Une application numérique sur la prothèse de hanche a été effectuée en utilisant la 
méthode hybride. Une comparaison entre deux formulations de la caractérisation du matériau de l'os a été effectuée pour relier les propriétés mécaniques de l'os. La structure optimale représente est plus fiable que la structure initiale et également en respectant un niveau cible de fiabilité. L'optimisation fiabiliste est donc une tache indispensable pour fournir des prothèses fiable et performantes.

\section{Références}

[BEA 90] BEAUPRÉ G.S., ORR T.E., CARTER D.R., « An approach for time-dependent bone modeling and remodelingapplication: A preliminary remodeling simulation », J. of Orthopaedic Research, vol. 8, n 5, p. 662-670, 1990.

[DOB 04] Doblare M., Garcia J.M., GoMEZ M.J., "Modelling bone tissue fracture and healing: A review », Engineering Fracture Mechanics, vol. 71, P. 1809-1840, 2004.

[KEL 94] KELLER T.S., « Predicting the compressive mechanical behavior of bone », J. of Biomech., vol. 27, p. 11591168, 1994.

[KHA 10] KhaRmanda G., El-HAmi A., SouZA DE CURSi E., « Reliability-Based Design Optimization », In edited book Multidisciplinary Design Optimization in Computational Mechanics, Edited by Piotr Breitkopt and Rajan Filomeno Coelho, Chapter 11, : Wiley \& Sons, April 2010, ISBN: 9781848211384 , Hardback 576 pp, 2010.

[KHA 15] KHARMANDA G., «Reliability analysis for cementless hip prosthesis using a new optimized formulation of yield stress against elasticity modulus relationship », Materials and Design (January 2015), vol. 65, p. 496-504, 2015.

[KHA 16] KHARMANDA, G., « Integration of multi-objective structural optimization into cementless hip prosthesis design: Improved Austin-Moore model », Computer Methods in Biomechanics and Biomedical Engineering, vol 19, n 14, p. 1557-1566, 2016. 